\title{
DETERMINACIÓN DE LAS CONDICIONES DE GERMINACIÓN Y EL EFECTO DE PRETRATAMIENTOS EN SEMILLAS DE CUPHEA GLUTINOSA (LYTHRACEAE)
}

\author{
FRANCISCO J. CARDINALI ${ }^{1,2}$, MARIO A. THEVENON ${ }^{1,3}$, MÓNICA L. MURCIA² y \\ MARÍA E. DI SANTO1
}

\begin{abstract}
Summary: Determination of germination conditions and the effect of pretreatments in Cuphea glutinosa seeds (Lythraceae). Cuphea glutinosa shows a high agronomic and agroindustrial potential. Germination conditions for this species are unknown. The objective of the work is to determine germination conditions, their impairments and the treatments to overcome them. There were utilized healthy seeds, complete and quite filled, gathered from a population of Sierra de los Padres, Buenos Aires province, Argentina. There were applied the following treatments, in six repetitions of 50 seeds, in a completely randomized design: blank, scarified, gibberellic acid, darkness, potassium nitrate, stratification and temperature alternation. The germination conditions after the pre-treatments were $24{ }^{\circ} \mathrm{C}(+/-1)$ under light, $100 \mu \mathrm{E} \cdot \mathrm{m}^{-2} \cdot \mathrm{sec}^{-1}$. The counts of emerged rootlets were performed at 6 and 12 days, except for the treatment with darkness, in which they were performed at 12 and 18 days. The effect of the treatments was evaluated by ANOVA and Tuckey tests $(p=0,05)$. The population showed $50 \%$ of germination; positive response to light, to stratification, and to potassium nitrate. There was no effect with scarification neither with temperature alternation. The gibberellic acid showed a negative effect in the percentage of germination, and affected the carbon partition during the growing of seedlings.
\end{abstract}

Key words: Dormancy, scarification, stratification, gibberellic acid, potassium nitrate, alternating temperatures, darkness.

Resumen: Cuphea glutinosa presenta alto potencial agroindustrial y agronómico. Se desconocen las condiciones de germinación para la especie. El objetivo del trabajo es contribuir a determinar condiciones de germinación, impedimentos para la misma y tratamientos para superarlos. Se utilizaron semillas sanas, enteras y llenas, recolectadas de una población de Sierra de los Padres, provincia de Buenos Aires, Argentina. Se aplicaron los siguientes tratamientos en 6 repeticiones de 50 semillas, bajo un diseño completamente aleatorizado: testigo, escarificado, ácido giberélico, oscuridad, nitrato de potasio, estratificación y alternancia de temperaturas. Las condiciones de germinación después de los pre tratamientos fueron a $24{ }^{\circ} \mathrm{C}(+/-1)$ con luz, $100 \mu \mathrm{E} \cdot \mathrm{m}^{-2} \cdot \mathrm{seg}^{-1}$. Los recuentos de radículas emergidas fueron a los 6 y a los 12 días, excepto en el tratamiento oscuridad en el que fueron a los 12 y a los 18 días. El efecto de los tratamientos se evaluó mediante ANOVA y Tuckey $(p=0,05)$. La población mostró $50 \%$ de germinación; respuesta positiva a la luz, a la estratificación y al nitrato de potasio. No tuvieron efecto el escarificado ni las temperaturas alternas. El ácido giberélico tuvo un efecto negativo en el porcentaje de germinación y afectó la partición de carbono durante el crecimiento de las plántulas.

Palabras clave: Dormición, escarificación, estratificación, ácido gibérelico, nitrato de potasio, alternancia de temperaturas, oscuridad.

\footnotetext{
${ }^{1}$ Lab. Botánica Dpto. Biología. FCEyN - UNMP. Funes 3250 (7600) Mar del Plata. Argentina. cardinal@mdp.edu.ar thevenon@mdp.edu.ar, mariedisanto@gmail.com

2 FCA - UNMP - EEAINTA Balcarce

${ }^{3}$ CNAI. UNMdP. Matheu 4051
} 


\section{INTRODUCCIÓN}

Cuphea glutinosa Cham. \& Schltdl. (Lythraceae), conocida vulgarmente como Siete Sangrías, es una especie originaria de América templado cálida. En Argentina se la encuentra habitualmente en la estepa clímax y en la región serrana (Cabrera \& Zardini, 1978; Marzocca, 1997), particularmente en las altiplanicies de las sierras pampeanas y subandinas de Córdoba, Catamarca, San Luis, Tucumán, Jujuy y Salta (Ratera \& Ratera, 1980).

Esta especie presenta un fruto tipo cápsula, que se abre a lo largo de una hendidura lateral, a través de la cual emergen las semillas. Al igual que todas las Lythraceae, produce un alto número de semillas por planta (Graham, 1989), entre 212 y 609 en una estación de crecimiento (Cardinali et al., 2009), con abundantes reservas de proteínas y en especial de lípidos, constituidos por ácidos grasos de cadena media (C8-C14) (Graham et al., 1981; Hirsinger, 1985). Ellos le aportan potencialmente un alto valor económico, dados sus múltiples usos en la agroindustria (Hirsinger \& Knowles, 1984; Thompson, 1984; Hirsinger, 1985). Asimismo en la nutrición, por su alto contenido en ácido láurico, actualmente suministrado casi exclusivamente por el coco y la médula de palmera (Graham, 1989; Knapp, 1993).

Dado el claro potencial agroindustrial de esta especie se vislumbra la posibilidad de su explotación agronómica, lo que pone de relevancia la importancia de los conocimientos para su cultivo. La germinación y los efectos de pretratamientos, como el frío o los activadores usados frecuentemente, como el ácido giberélico y el nitrato de potasio, son aspectos totalmente desconocidos para la especie.

El objetivo del presente trabajo es contribuir a determinar las condiciones más adecuadas para evaluar la germinación en $C$. glutinosa, los factores que constituyen posibles impedimentos para la germinación y los pretratamientos requeridos para superarlos.

\section{Material y Método}

El estudio se realizó sobre semillas producidas por una población de C. glutinosa de Sierra de

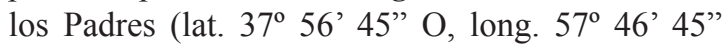
S), sistema de Tandilia, Buenos Aires, Argentina. Las plantas fueron identificadas mediante claves taxonómicas según Cabrera \& Zardini (1978). Se recolectaron semillas maduras y enteras, a partir de cápsulas en dehiscencia, durante los meses de febrero y marzo de 2012. Se clasificaron por tamaño, se descartaron las más pequeñas y se seleccionaron aquellas sanas enteras y visiblemente llenas, las que resultaron con un peso de mil semillas de 1,07 gramos (+/- 0,06).

A efecto de determinar la capacidad germinativa de las semillas recolectadas, dado que no hay información específica para C. glutinosa, se efectuó la siembra en sustrato papel húmedo (Fig. 1), sugerido para semillas pequeñas, con adecuada humedad y aireación, acorde a la metodología básica de la Asociación Internacional de Ensayos de Semillas (ISTA, 2008).

Cada uno de los 7 tratamientos contó con 6 repeticiones de 50 semillas, que fueron ubicadas en cajas de Petri plásticas de $9 \mathrm{~cm}$ de diámetro con doble papel de filtrado medio. Las cajas se dispusieron siguiendo un diseño completamente aleatorizado en estufa de cultivo a $24{ }^{\circ} \mathrm{C}(+/-1)$, con puerta de vidrio transparente que recibía luz del entorno a una intensidad de $100 \mu \mathrm{E} \cdot \mathrm{m}^{-2}$. $\mathrm{seg}^{-1}$. La capacidad germinativa $(\mathrm{G})$ de las semillas se evaluó como porcentaje, efectuando recuentos de radículas emergidas a los 6 y a los 12 días.

A continuación se describen los detalles de cada tratamiento:

1) Testigo. Se aplicó el protocolo básico hidratando con $8 \mathrm{ml}$ de agua desmineralizada (Fig. 1).

2) Escarificado. La semillas sufrieron un leve desgaste de sus cubiertas por escarificado suave con lija seca 326U3M, (Fig. 1 y 2). Luego se aplicó el protocolo básico.

3) Ácido giberélico $\left(\mathrm{GA}_{3}\right)$. Se aplicó el protocolo básico hidratando con $8 \mathrm{ml}$ de una solución acuosa de 60 ppm de $\mathrm{GA}_{3}$, (Fig. 1).

4) Oscuridad. Las cajas fueron sometidas al protocolo básico pero cubiertas completamente con papel de aluminio durante 6 días. Luego de este período se removieron las coberturas posibilitando la llegada de luz a la semilla. Se registró el nivel de germinación a los 12 días y más tarde a los 18 días para uniformar los tiempos del proceso de germinación con exposición a $100 \mu \mathrm{E} \cdot \mathrm{m}^{-2} \cdot \mathrm{seg}^{-1}$, (Fig. 1).

5) Nitrato de potasio $\left(\mathrm{NO}_{3} \mathrm{~K}\right)$. Se aplicó el protocolo básico hidratando con $8 \mathrm{ml}$ de una solución acuosa de nitrato de potasio al $0,2 \%$. 


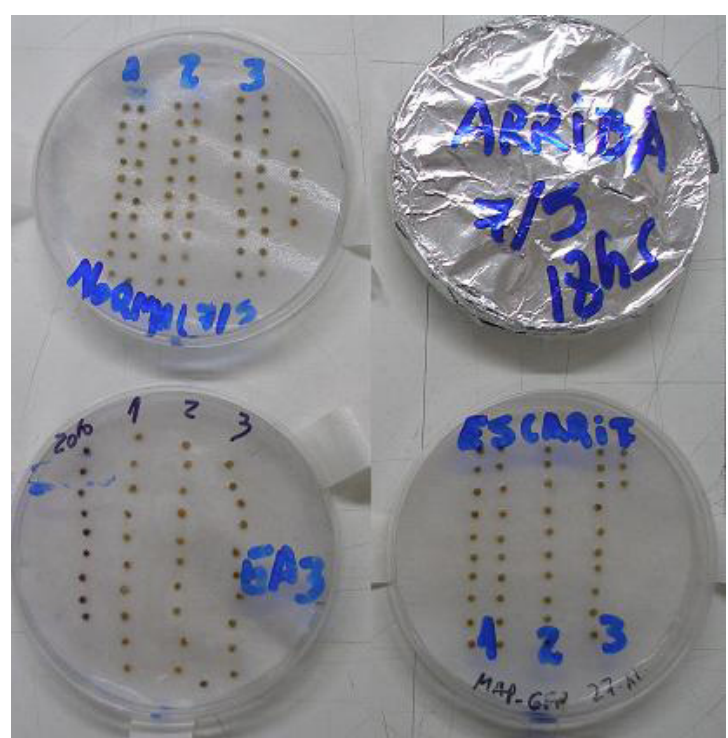

Fig. 1. Modelo experimental de siembra en sustrato papel húmedo mostrando algunos de los tratamientos, acorde a la metodología básica de la Asociación Internacional de Ensayos de Semillas (ISTA, 2008).

6) Estratificación. Las semillas fueron sembradas como en el tratamiento testigo y ubicadas en heladera a $5{ }^{\circ} \mathrm{C}$ para el tratamiento con baja temperatura. Al cabo de 8 días fueron transferidas a estufa de cultivo siguiendo el protocolo básico.

7) Alternancia de temperaturas. Se aplicó el protocolo básico pero en cámara de crecimiento provista de 6 tubos fluorescentes de 18 watts. La temperatura durante el período lumínico fue de $30{ }^{\circ} \mathrm{C}(+/-2)$ y durante el oscuro fue de $20{ }^{\circ} \mathrm{C}$ $(+/-2),\left(30^{\circ} \mathrm{C}, \mathrm{L}, 12 \mathrm{~h}: 20^{\circ} \mathrm{C}, \mathrm{O}, 12 \mathrm{~h}\right)$.

El efecto de los tratamientos realizados en bloques completamente aleatorizados se evaluó mediante ANOVA y las medias fueron comparadas mediante el test de Tuckey $(p=0,05)$.

\section{Resultados Y DISCUSIÓN}

La metodología básica de siembra aplicada fue satisfactoria en lo referido al sustrato, nivel de humedad y temperatura en los que se desarrolló el trabajo. La temperatura aplicada $\left(24{ }^{\circ} \mathrm{C}+/-1\right)$ es coincidente con la temperatura óptima de entre 18 y

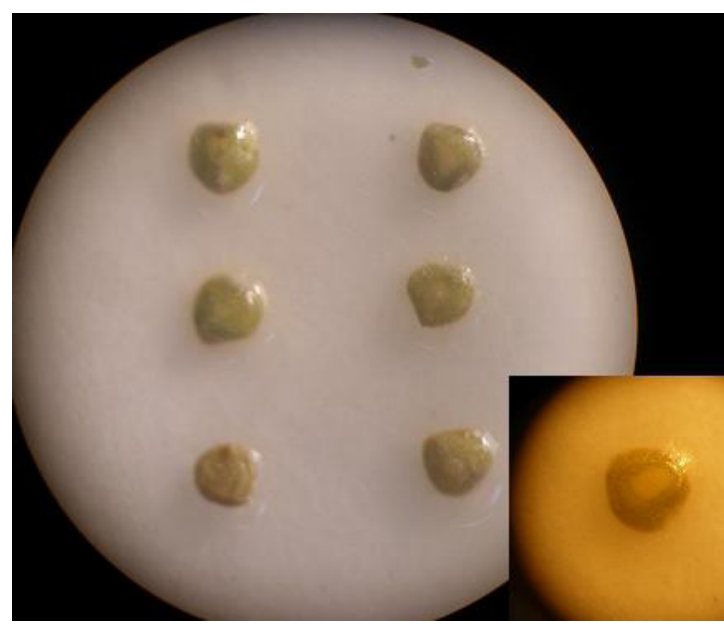

Fig. 2. Vista del tratamiento escarificado y detalle de una semilla con el desgaste de sus cubiertas por escarificado suave con lija seca 326U3M.

$24{ }^{\circ} \mathrm{C}$ determinada por Berti y Johnson (2008) para germinación de Cuphea viscosissima Jacq.

Los resultados de los tratamientos aplicados se presentan en la Figura 3.

1) Testigo. Permitió determinar que el nivel de germinación $(\mathrm{G})$ de las semillas producidas por la población natural de Sierra de los Padres fue del $50 \%$. Estos resultados indicarían que la mitad de las semillas producidas no germinan por algún tipo de impedimento causante de dormición o simplemente por falta de viabilidad. Si bien no hay datos de esta especie en particular, Roath et al. (1994) determinó para C. viscosissima valores muy variables del porcentaje de dormición entre las numerosas plantas muestreadas, el cual fluctuó entre el 7,06 y el 77\%, atribuido a la dormición generada principalmente por las cubiertas y en menor medida por los embriones.

Si bien no se cuantificó el porcentaje de plántulas normales se observó un desarrollo adecuado de hipocótilos y cotiledones de las plántulas obtenidas al finalizar el ensayo.

2) Escarificado. Este tratamiento que eventualmente permitiría superar los impedimentos ocasionados por las cubiertas de la semilla, no arrojó diferencias significativas con respecto al testigo. Las semillas de C. glutinosa poseen una cubierta dura debido a la presencia de una 


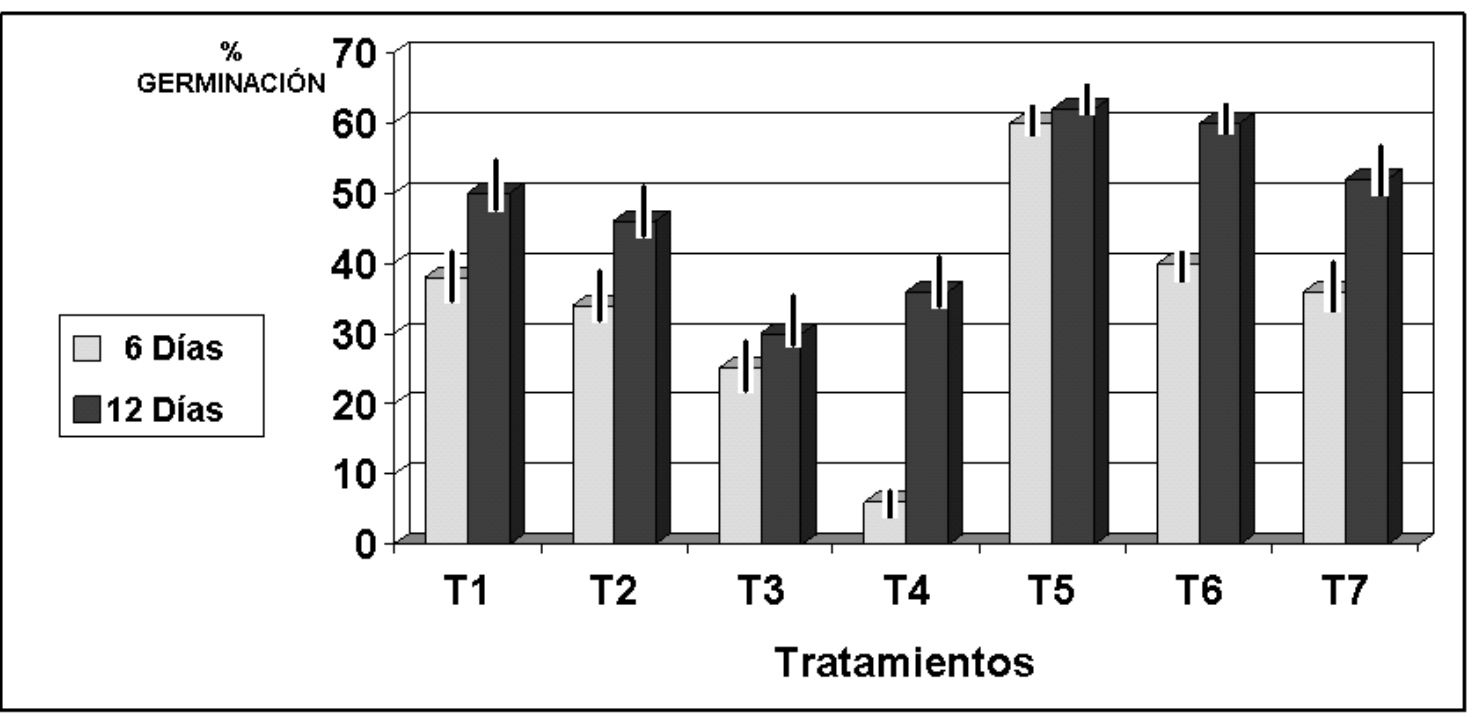

Fig. 3. Resultados de los diferentes tratamientos expresados en porcentaje de germinación a los 6 y 12 días. ANVA y CV p=0,05. (T1: Testigo; T2: Escarificado; T3: GA3 (60ppm); T4: Oscuridad; T5: NO3K (0,2\%); T6: Estratificación; T7: Alternancia de temperaturas).

endotesta de células esclerenquimáticas que van siendo sustituidas por células parenquimáticas hacia el extremo más aguzado de la semilla. Asimismo, el tegumento interno o tegmen muestra varias capas de células parenquimáticas (exotegmen) y una capa de macroesclereidas (mesotegmen) o Capa de Malpighi (Cardinali, et al. 2010). Si bien estas capas de células, de paredes fuertemente engrosadas que incluyen la presencia de lignina, le confieren resistencia mecánica, evidentemente no constituyen una barrera absoluta para la hidratación. Es probable que el ingreso de agua a la semilla ocurra por el extremo aguzado de la misma, constituido sólo por células parenquimáticas. En consecuencia, las cubiertas no serían responsables de una dormición primaria. Si bien no hay reportes científicos sobre escarificación en C. glutinosa, estos datos se oponen a los escasos resultados citados por la bibliografía para el género Cuphea. Así, Widrlechner \& Kovach (2000) indican que algunas especies de Cuphea exhiben dormición primaria, la cual generalmente está asociada a la dureza de las cubiertas. Coincidentemente, Roath et al. (1994) destacan la dormición impuesta por las cubiertas en C. viscosissima, la cual fue verificada hasta 5 años después de cosechada la semilla.
3) Ácido giberélico. Las giberelinas, en particular el $\mathrm{GA}_{3}$, son ampliamente utilizadas para promover o inducir la geminación de semillas en diversas especies (Ikuma \& Thimann, 1963; Tigabú \& Odén, 2001) y romper la dormición permitiendo la germinación y crecimiento del embrión (Siobhan \& McCourt, 2003).

Contrariamente en este trabajo la aplicación de una solución acuosa de 60 ppm de GA 3 causó una depresión en el porcentaje de germinación. Estos resultados son plenamente coincidentes con los obtenidos por Amador-Alférez et al. (2013) quienes, al trabajar en Ferocactus histrix (DC.) G.E. Linds. (Cactaceae), tuvieron porcentajes de germinación en el noveno día de 38,$6 ; 37,4$ y 33,8 para concentraciones de 125 , 250 y $500 \mathrm{ppm}$ de $\mathrm{GA}_{3}$ respectivamente, en contraste con el $56,7 \%$ del testigo. Estos resultados pueden ser atribuidos a que el $\mathrm{GA}_{3}$ agregado exógenamente se sumaría a la concentración endógena, produciendo un efecto inhibidor de la síntesis de GA endógena por retroalimentación negativa, tal como lo menciona Avila Alejandre (2003) para Zea mays L. (Poaceae).

Respecto a estas observaciones, cabe aclarar que no hay reportes sobre este aspecto para $C$. glutinosa, sin embargo Widrlechner \& Kovach (2000) al hidratar con una solución acuosa de $1 \mathrm{mM} \mathrm{GA}_{3}$ (equivalente a 346 ppma) semillas de $C$. angustifolia 


\section{F. J. Cardinali et al. - Semillas de Cuphea glutinosa}

Jack. Ex Koehne, C. carthagenensis (Jacq.) J.F. Macbr., C. koehneana Rose, C. lutea Rose ex Koehne, C. painteri Rose ex Koehne, C. paucipetala S.A. Graham, C. procunbens Ortega, C. tolucana Peyr. y C. wrightii A. Gray, sólo tuvieron respuesta significativa en C. procumbens.

Si bien, en el presente trabajo, el GA 3 provocó una disminución de $\mathrm{G}$, se pudo observar un notable efecto sobre el crecimiento del hipocótilo y de la radícula (Fig. 4), aún al aplicar concentraciones menores a las utilizadas por los autores antes citados.

Los efectos consistieron en una fuerte alteración de la partición de carbono de las plántulas. En el primer recuento, a los 6 días, se observaron hipocótilos alargados y delgados que duplicaron en longitud al testigo. Este efecto se vio moderado en el segundo recuento, aunque se mantuvo una significativa diferencia (Tabla1).

Tabla 1. Longitud del hipocótilo y variaciones en porcentaje de las plántulas testigo y de las tratadas con $\mathrm{GA}_{3}$ a los 6 y 12 días.

\begin{tabular}{|lcc|}
\hline \multicolumn{3}{|c|}{ Longitud del Hipocótilo $(\mathrm{mm})$} \\
\hline & Testigo & GA $_{3}$ \\
\hline días & 8,41 & 16,25 \\
12 días & 13,30 & 22,00 \\
Incremento $(\%)$ & 58,15 & 35,38 \\
\hline
\end{tabular}

El notable crecimiento del hipocótilo repercutió, como fenómeno fisiológico de correlación, en el crecimiento de la radícula. En efecto, el ajuste de esta alteración carbonada se refleja en una importante disminución del diámetro radicular, pero sin alterar su longitud. Esto está indicando que se prioriza el mantenimiento del alargamiento radicular y en consecuencia su capacidad de exploración, siendo la variable de ajuste del crecimiento el espesor de la radícula. Este efecto se manifiesta claramente a través de las relaciones hipocótilo-radícula (Tabla 2).

\section{Tabla 2. Longitudes y diámetros de hipocótilo y radícula expresados en milímetros de las plántulas testigo y de las tratadas con $\mathrm{GA}_{3}$ y sus relaciones a los 12 días.}

\begin{tabular}{|lcccc|}
\hline \multicolumn{4}{c}{ Tratamiento } \\
& \multicolumn{3}{c|}{ Hipocótilos } & \multicolumn{2}{c|}{ Radícula } \\
& Testigo & GA $_{3}$ & Testigo & GA $_{3}$ \\
Longitud & $13,30 \mathrm{a}$ & $22,00 \mathrm{~b}$ & $7,96 \mathrm{a}$ & $8,34 \mathrm{a}$ \\
Relación & $0,60: 1$ & $0,95: 1$ \\
Diámetro & $0,559 \mathrm{a}$ & $0,503 \mathrm{a}$ & 0,609 a & $0,298 \mathrm{~b}$ \\
Relación & \multicolumn{2}{c}{$1,11: 1$} & \multicolumn{2}{c}{$2,04: 1$} \\
\hline
\end{tabular}

*letras iguales indican diferencias no significativas entre tratamientos

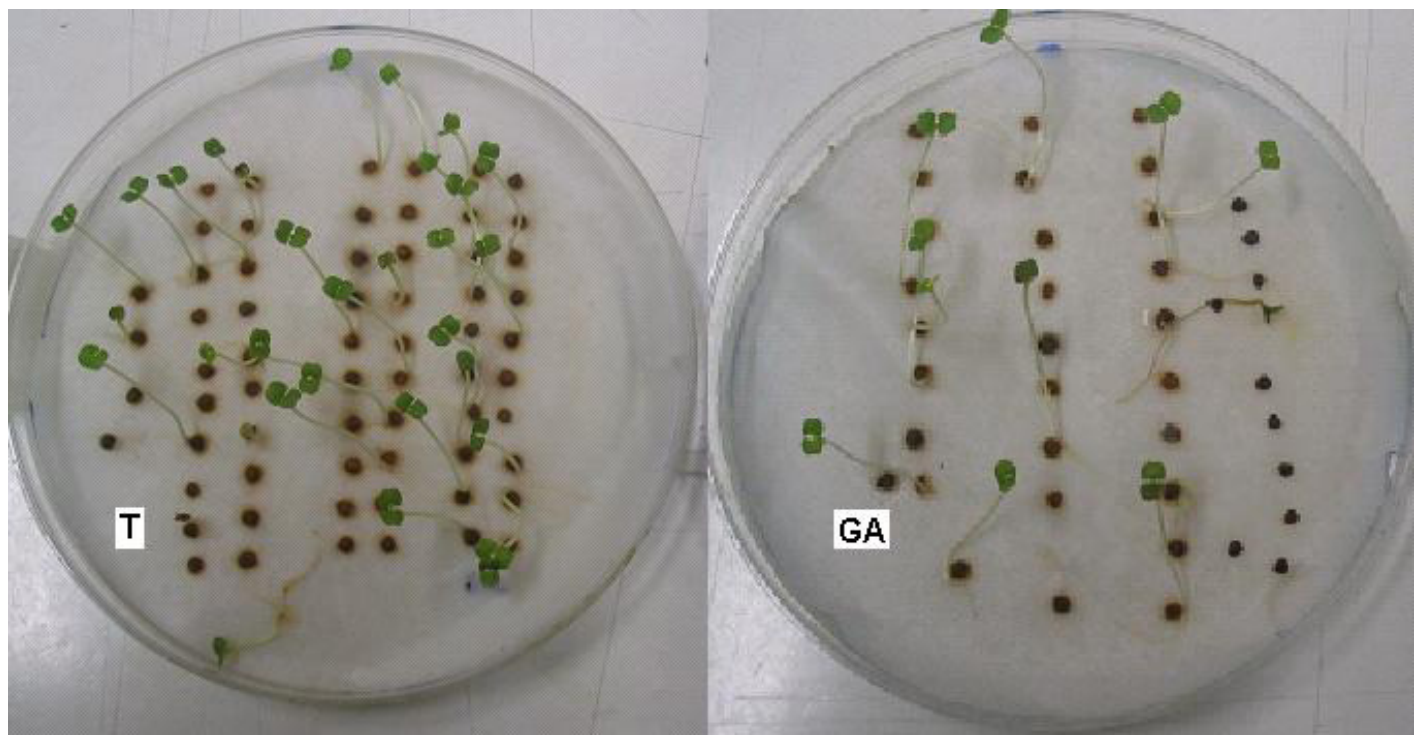

Fig. 4. Semillas germinadas correspondientes a los tratamientos T: testigo y GA: Ácido giberélico. 
4) Oscuridad. Este tratamiento puso claramente en evidencia la necesidad de luz para la germinación, ya que en oscuridad absoluta el nivel de germinación alcanzó sólo el 6\%, lo que representa el 15,79\% respecto del testigo. La posterior exposición a la luz permitió alcanzar en promedio el 36\% a los 12 días y el $58 \%$ a los 18 días, valores estadísticamente semejantes al testigo. Estos resultados indican claramente que $C$. glutinosa requiere luz para germinar, es decir es una semilla fotoblástica positiva. Si bien no hay datos específicos para esta especie, estos resultados coinciden con lo observado en C. viscosisima por Widrlechner \& Kovach (2000), quienes demostraron la necesidad de luz para alcanzar los máximos niveles de germinación en los tratamientos de frío. Asimismo, Hernández Mendoza \& Parra Tabla (2013), al trabajar con $C$. gaumeri Koehne reportaron una respuesta positiva a la luz consistente en una mayor velocidad de germinación.

5) Nitrato de potasio. El nitrato de potasio es un promotor de la geminación recomendado ampliamente en las Reglas Internacionales de Ensayos de Semillas (ISTA, 2008) para superar la dormición en semillas de diversas especies. Asimismo, la bibliografía es abundante en cuanto a resultados favorables por el uso de este compuesto en semillas con dificultades para germinar. En este trabajo la hidratación con solución acuosa de nitrato de potasio permitió alcanzar el máximo porcentaje final de germinación. Sin embargo, lo más relevante es el considerable aumento de la velocidad de germinación, ya que en el primer recuento se alcanzaron porcentajes de germinación muy superiores a los del testigo y al tratamiento estratificado, que también resultó positivo, y se lograron prácticamente los máximos valores de germinación en el recuento final.

Si bien no hay reportes específicamente para C. glutinosa, Widrlechner \& Kovach (2000) trabajando con 9 especies de Cuphea encontraron respuesta positiva en C. koehneana, C. procunbens, C. tolucana, C. carthagenensis y C. lutea, al aplicar idénticas concentraciones de nitrato de potasio que en el presente trabajo. Como información adicional estos autores reportan la comunicación personal de A. Logan-Miller, National Seed Storage Laboratory, acerca de una respuesta positiva de semilla de Cuphea a la aplicación de nitrato de potasio sólo con previo almacenamiento durante cierto período de tiempo.

6) Estratificación. En este trabajo se encontró una significativa respuesta al frío luego de 8 días a $5{ }^{\circ} \mathrm{C}$, en tanto que los resultados logrados por Widrlechner \& Kovach (2000) para C. viscosissima indican la necesidad de aplicar 16 a 24 semanas de tratamiento a $4{ }^{\circ} \mathrm{C}$ y en presencia de luz para alcanzar niveles superiores al 80\% de germinación; en tanto que para alcanzar niveles de germinación similares a los logrados en este trabajo (60\%) debieron aplicar entre 4 y 8 semanas de frío. La rápida respuesta al pretratamiento a $5{ }^{\circ} \mathrm{C}$ por parte de $C$. glutinosa, muestra claramente la efectividad para desencadenar el proceso de germinación. No obstante ello, durante el tratamiento a baja temperatura y en presencia de luz, ninguna de las semillas presentó salida de radícula, indicadora de germinación, lo que permite inferir que $C$. glutinosa tiene un umbral térmico de germinación superior a $\operatorname{los} 5^{\circ} \mathrm{C}$.

Estos resultados se corresponden con los aportes de Berti \& Johnson (2008) para C. viscosissima, quienes encontraron que la temperatura mínima constante para la germinación varió entre 6 y $10^{\circ} \mathrm{C}$.

En general las respuestas a la estratificación están relacionadas a la caída en la concentración de acido abscísico (ABA), a un incremento en los promotores - básicamente giberelinas - o a ambos efectos simultáneamente. Sondheimer et al. (1974) observaron una rápida metabolización del ABA por efecto de la estratificación a $5{ }^{\circ} \mathrm{C}$ de embriones de Fraxinus americana L. (Oleaceae). Asimismo, Al-Imam \& Qrunfleh (2007) demostraron que la estratificación de semillas de Prunus amygdalus L. (Rosaceae) determinó una significativa disminución del ABA endógeno libre y ligado durante los primeros 15 días y una completa declinación a los 45 días. En este trabajo no hay respuesta a $\mathrm{GA}_{3}$, de manera que según las evidencias generales sería posible considerar que la respuesta a este tratamiento es debida a una caída en la concentración de ABA o eventualmente al aumento de otras giberelinas.

7) Alternancia de temperaturas. La alternancia de temperaturas no produjo ninguna mejora en el porcentaje ni en la velocidad de germinación, ya que los resultados obtenidos no difieren de los alcanzados por el tratamiento testigo. Estos 
resultados coinciden con Widrlechner \& Kovach (2000) que trabajaron con semillas en dormición de C. viscosisima, $y$ no tuvieron respuesta al tratamiento con alternancia de temperatura de $30 / 20{ }^{\circ} \mathrm{C}$ y en presencia de luz. Estos resultados se mantuvieron aún con tratamientos adicionales de $\mathrm{GA}_{3}$ o nitrato de potasio. Opuestamente, Rosa y Ferreira (1998) reportaron que semillas de C. carthagenensis alcanzaron más del $80 \%$ de germinación bajo alternancia de temperaturas y en cambio a temperaturas constantes de $10^{\circ} \mathrm{C} ; 15^{\circ} \mathrm{C}$; $20^{\circ} \mathrm{C} ; 25^{\circ} \mathrm{C} ; 30^{\circ} \mathrm{C}$ y $35^{\circ} \mathrm{C}$ germinaron en un muy bajo porcentaje aunque las semillas permanecieron viables.

\section{Conclusiones}

La población natural de Cuphea glutinosa de Sierra de los Padres mostró semillas con un nivel de germinación del 50\%. Si bien las semillas presentan cubiertas muy duras, el tratamiento escarificado no mejoró el porcentaje de germinación, lo que estaría indicando que no hay un impedimento en el ingreso de agua ni una resistencia mecánica para la emergencia de la radícula. La aplicación exógena de una solución acuosa de 60 ppm de $\mathrm{GA}_{3}$ causó una depresión en el porcentaje de germinación y alteró la partición de carbono durante el crecimiento de la plántula. La alternancia de temperaturas no tuvo efectos en el porcentaje de germinación. Sin embargo, se observó claramente que la estratificación de las semillas aumentó significativamente el porcentaje de germinación y que el umbral mínimo de temperatura para la germinación es superior a los $5^{\circ} \mathbf{C}$. Los resultados dieron cuenta que las semillas de esta especie se comportaron como fotoblásticas positivas y respondieron aumentando significativamente el porcentaje y la velocidad de germinación, cuando fueron tratadas con $\mathrm{NO}_{3} \mathrm{~K}$.

\section{Agradecimientos}

Este trabajo está enmarcado en el proyecto: "Estudio integral de especies medicinales autóctonas: Cuphea glutinosa Cham. et Schldtl. nativa de las sierras bonaerenses" Exa-UNMdP $\mathbf{N}^{\circ}$ 655/14, subsidiado por la Universidad Nacional de
Mar del Plata, que se desarrolla en el Laboratorio de Botánica de la Facultad de Ciencias Exactas y Naturales, Universidad Nacional de Mar del Plata, República Argentina.

\section{Bibiliografía}

AL-IMAM, N. M. A. \& M. M. QRUNFLEH. 2007. Physiological study of the stratification effect on the dynamic changes in endogenous abscisic acid in bitter almond seeds (Prunus amygdalus) and its relation to seeds germination. Mesopotamia J. Agric. 35: $1-9$.

AMADOR-ALFÉREZ, K. A., J. DÍAZ-GONZÁLEZ, S. LOZA-CORNEJO \& E. Y. BIVIÁNCASTRO. 2013. Efecto de diferentes reguladores de crecimiento vegetal sobre la germinación de semillas y desarrollo de plántulas de dos especies de Ferocactus (Cactaceae). Polibotánica 35: 109-135.

AVILA ALEJANDRE, A. X. 2003. Regulación por retroalimentación de la biosíntesis de giberelinas en semillas de Zea mays L. Tesis, Universidad Autónoma Metropolitana, Iztapalapa.

BERTI, M. T. \& B. L. JOHNSON. 2008. Seed germination response of Cuphea to temperature. Industr. Crops Prod. 27: 17-21.

CABRERA, A. L. \& E. M. ZARDINI. 1978. Manual de la Flora de los alrededores de Buenos Aires. Editorial Acme, Buenos Aires.

CARDinAli, F. J., M. E. DI SANTO \& M. A. THEVENON. 2009. Secuencia de la floración y la fructificación y calidad de semilla en Cuphea glutinosa Cham et Schltdl. (Lythraceae). Actas de XXXII Jornadas Argentinas de Botánica, Huerta Grande, Córdoba.

CARDINALI, F. J., M. A. THEVENON \& M. E. ARIAS. 2010. Estudio morfoanatómico de la semilla y de las reservas proteicas y lipídicas en tejidos cotiledonales de Cuphea glutinosa (Lythraceae). Bol. Soc. Argent. Bot. 45: 47-55.

GRAHAM, S. A., F. HIRSINGER \& G. ROBBELEN. 1981. Fatty acids of Cuphea (Lythraceae) seed lipids and their systematic significance. Amer. J. Bot. 68: $908-917$.

GRAHAM, S. A. 1989. Cuphea: a new plant source of medium-chain fatty acids. Crit. Rev. Food Sci. Nutr. 28: 139-173.

HERNÁNDEZ MENDOZA, V. B. \& V. PARRA TABLA. 2013. Efectos de agua y luz en la adecuación de la especie endémica Cuphea gaumeri (Lythraceae). IV Congreso Mexicano de Ecología, Villa Hermosa, Tabasco.

HIRSINGER, F. 1985. Agronomic potential and seed composition of Cuphea, an annual crop for lauric 
and capric seed oil. J. Amer. Oil Chem. Soc. 62:7680.

HIRSINGER, F. \& P. F. KNOWELES. 1984. Morphological and agronomic description of selected Cuphea germplasm. Econ. Bot. 38: 439451.

IKUMA, H. \& K. V. THIMANN. 1963. Action of Kinetin on photosentitive germination of lettuce seed as compared with that of gibberellic acid. Plant Cell Physiol. 4: 113-128.

ISTA. 2008. International Rules for Seed Testing. International Seed Testing Association, Bassersdorf.

KNAPP, S. J. 1993. Breakthroughs towards the domestication of Cuphea. In: J. Janick and J. E. Simon (eds.), New Crops, pp. 372-379. Wiley, New York.

MARZOCCA, A. 1997. Vademécum de malezas medicinales de la Argentina indígenas y exóticas. Orientación Gráfica Editora, Buenos Aires.

RATERA, L. R. \& M. O. RATERA. 1980. Plantas de la flora argentina empleadas en medicina popular. Hemisferio Sur, Buenos Aires.

ROATH, W. W., M. P. WIDRLECHNER \& R. KLEIMAN. 1994. Variability in Cuphea viscosissima Jacq. Collected in east-central United States. Industr. Crops Prod. 3: 217-223.
ROSA S. G. T. \& A. G. FERREIRA. 1998. Germinação de sementes de espécies medicinais do Rio Grande do Sul: Bromélia antiacantaha Bert., Cuphea carthagenensis (Jacq.) Macbride e Talinum patens (Jacq.) Willdenow. Acta Bot. Bras. 12, suppl.1: 515-522.

SIOBHAN, M. B. \& P. MCCOURT. 2003. Hormone Cross-Talk in Seed Dormancy. J. Plant Growth Regul. 22: 25-31.

SONDHEIMER, E., E. C. GALSO, E. TINELLI \& D. C. WALTON. 1974. The metabolism of hormones during seed germination and dormancy. Plant Physiol. 54: 803-808.

THOMPSON, A. E. 1984. Cuphea a potential new crop. Hort. Science 19: 352-354.

TIGABU, M. \& P. C. ODEN. 2001. Effect of scarification, gibberellic acid and temperature on seed germination of two multipurpose Albizia species from Ethiopia. Seed Sci. Technol. 29: 11-20.

WIDRLECHNER, M. P. \& D. A. KOVACH. 2000. Dormancy-breaking protocols for Cuphea seed. Seed Science Technol. 28: 1-27.

Recibido el 21 de octubre de 2014, aceptado el 14 de abril de 2015 . 